\title{
TYK2 is a key regulator of the surveillance of B lymphoid tumors
}

\author{
Dagmar Stoiber, ${ }^{1}$ Boris Kovacic, ${ }^{1}$ Christian Schuster, ${ }^{1}$ Carola Schellack, ${ }^{1}$ Marina Karaghiosoff, ${ }^{2}$ \\ Rita Kreibich,1 Eva Weisz,1 Michaela Artwohl, ${ }^{1}$ Olaf C. Kleine, ${ }^{1}$ Mathias Muller, ${ }^{2}$ \\ Sabina Baumgartner-Parzer, ${ }^{3}$ Jacques Ghysdael, ${ }^{4}$ Michael Freissmuth, ${ }^{1}$ and Veronika Sexl ${ }^{1}$
}

1Department of Pharmacology, Medical University of Vienna, Vienna, Austria. ${ }^{2}$ Institute of Animal Breeding and Genetics, Veterinary University of Vienna, Vienna, Austria. ${ }^{3}$ Department of Endocrinology, Medical University of Vienna, Vienna, Austria. ${ }^{4}$ 'Unité Mixte de Recherche 146 CNRS/Institut Curie, Orsay, France.

\begin{abstract}
Aberrant activation of the JAK-STAT pathway has been implicated in tumor formation; for example, constitutive activation of JAK2 kinase or the enforced expression of STAT5 induces leukemia in mice. We show here that the Janus kinase TYK2 serves an opposite function. Mice deficient in TYK2 developed Abelsoninduced B lymphoid leukemia/lymphoma as well as TEL-JAK2-induced T lymphoid leukemia with a higher incidence and shortened latency compared with WT controls. The cell-autonomous properties of Abelson murine leukemia virus-transformed (A-MuLV-transformed) $\mathrm{TYK2}^{-/-}$cells were unaltered, but the high susceptibility of $\mathrm{TYK2}^{-/-}$mice resulted from an impaired tumor surveillance, and accordingly, TYK2 ${ }^{-/-}$ A-MuLV-induced lymphomas were easily rejected after transplantation into WT hosts. The increased rate of leukemia/lymphoma formation was linked to a decreased in vitro cytotoxic capacity of TYK2 ${ }^{-/-} \mathrm{NK}$ and NKT cells toward tumor-derived cells. RAG2/TYK2 double-knockout mice succumbed to A-MuLV-induced leukemia/lymphoma faster than $\mathrm{RAG2}^{-/-} \mathrm{TYK2}^{+/-}$mice. This defines $\mathrm{NK}$ cells as key players in tumor surveillance in Abelson-induced malignancies. Our observations provide compelling evidence that TYK2 is an important regulator of lymphoid tumor surveillance.
\end{abstract}

\section{Introduction}

The JAK-STAT pathway regulates cell proliferation, differentiation, and survival in hematopoietic cells $(1,2)$. Binding of a cytokine to its cognate receptor activates receptor-associated JAKs which in turn mediate the subsequent tyrosine phosphorylation of STAT proteins. Phosphorylated STAT proteins form dimers, translocate to the nucleus, and bind to specific DNA elements to induce or modulate expression of target genes. Gene knockout studies in mice underlined the vital role of the JAK-STAT pathway for hematopoiesis and other developmental processes (3).

Recent interest had focused on the role of the JAK-STAT pathway in tumor formation. Aberrant activation of JAK-STAT signaling had been shown in multiple solid tumors and leukemia (4-9). In particular, STAT3 and STAT5 have drawn much attention; both transcription factors display oncogenic properties when expressed ectopically. A constitutively active mutant of STAT3 was shown to transform rat fibroblasts (10), and the enforced expression of WT STAT5 in the lymphoid lineage induced $\mathrm{T}$ cell leukemia in mice (11). In addition, JAK1 has been implicated in transformation by the $v$-abl as well as by the $v$-src oncogene $(12,13)$, and constitutive activation of JAK2 as in the TEL-JAK2 oncogene suffices to induce leukemia in mice and humans $(14,15)$. On the other hand, JAK1 was shown to be a tumor suppressor downstream of IFN- $\gamma$ in a B lymphoid tumor model (16). A role as tumor suppressor downstream of IFN- $\gamma$ has also been attributed to STAT1 (17-21). STAT1 $1^{--}$mice have been repeatedly used to study the role of IFN- $\gamma$ in tumorigenesis

Nonstandard abbreviations used: A-MuLV, Abelson murine leukemia virus; $\mathrm{EC}_{50}$, $50 \%$ effective concentration; MTT, 3-(4,5-dimethylthiazol-2-yl)-2,5-diphenyltetrazolium bromide; NKT cell, NK T cell.

Conflict of interest: The authors have declared that no conflict of interest exists.

Citation for this article: J. Clin. Invest. 114:1650-1658 (2004).

doi:10.1172/JCI200422315. and tumor surveillance $(18,19)$. Recent evidence also proposed that STAT1 acted as a tumor suppressor by modulating apoptosis (22).

TYK 2 has been initially defined as a mediator of IFN- $\alpha / \beta$ signaling (23). TYK2 is also activated in response to several cytokines, including IL-6, IL-10, IL-12, and IL-13 (24). Studies with knockout mice have confirmed the essential role of TYK2 in host defense: TYK2-deficient mice are viable and fertile and show no obvious phenotype unless they are challenged with an excessive virus load $(25,26)$. Lack of TYK2 impaired type I IFN and IL-12 signaling but, unexpectedly, also some aspects of IFN- $\gamma$ signaling. In addition, TYK2 deficiency led to resistance against LPSinduced endotoxin shock (27).

Based on the available evidence, it is difficult to predict a role for TYK2 in tumor formation. To explore the function of TYK2 in the formation of B lymphoid leukemia, we used the Abelson-induced tumor model. The single exposure to the Abelson murine leukemia virus (A-MuLV) induces a slowly evolving B lymphoid leukemia/lymphoma that mimics multistep tumorigenesis (28). The $v$-abl gene is a hybrid of the viral gag gene fused with a sequence derived from the endogenous $c$-abl gene and encodes a nonreceptor nuclear tyrosine kinase (29). In people, $a b l$ is activated by a chromosomal translocation $(9 ; 22)$ that results in the so-called Philadelphia chromosome. The resulting fusion protein, bcr-abl, has constitutive kinase activity and is associated with chronic myeloid leukemia and acute lymphoid leukemia $(30,31)$.

Our study shows that TYK2-/- mice are tumor prone and links the increased tumor susceptibility to tumor surveillance. We thereby define TYK2 as a key molecule in B lymphoid malignancy.

\section{Results}

Increased frequency of Abelson-induced B cell lenkemia/lymphoma in TYK2 $2^{--}$mice. We were interested in whether deletion of TYK2 would modulate the onset and outcome of lymphoid malignancies. Infec- 

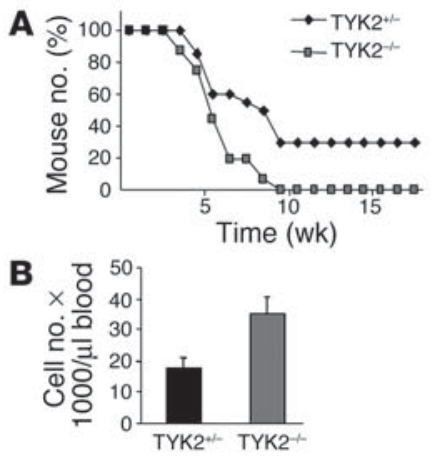
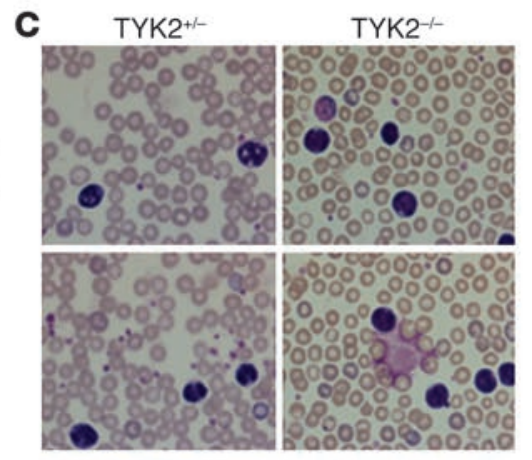
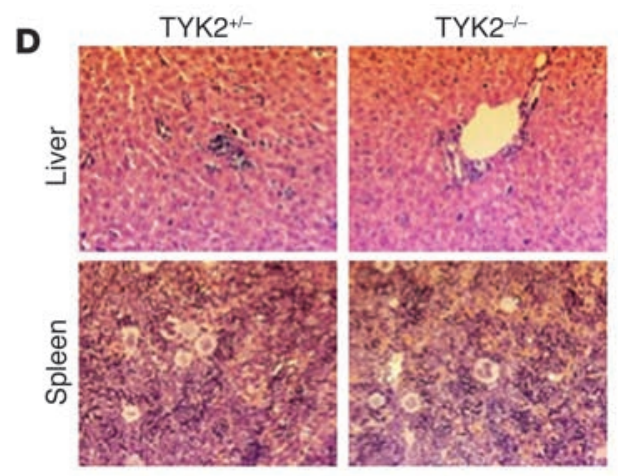

\section{Figure 1}

Effect of in vivo oncogenic challenge with the Abelson oncogene. (A) Kaplan-Meier plot of TYK2 ${ }^{+/-}$and TYK2 $2^{-/-}$mice after injection of a replication-incompetent A-MuLV retrovirus ( $n=20$ for each group). All 20 TYK2 ${ }^{-/-}$mice died of leukemia/lymphoma within 10 weeks, whereas 6 TYK2 ${ }^{+/-}$mice remained disease free for more than 6 months. (B) White blood cell count of the diseased TYK2 $2^{+/-}$and TYK2 $2^{-/-}$animals $(n=8$ for TYK2 $^{-/-}$and $n=15$ for TYK2 ${ }^{+-}$). (C) H\&E-stained blood smears of TYK2 ${ }^{+--}$and TYK2 ${ }^{-/-}$mice (magnification, $\left.\times 200\right)$. Typical examples of each genotype are shown. (D) H\&E-stained histological sections (magnification, $\times 200$ ) of liver (upper panels) and spleen (lower panels) of TYK2 ${ }^{+/-}$and TYK2 ${ }^{-1-}$ animals. One representative example is depicted.

tion of newborn mice with a replication-deficient, ecotropic form of A-MuLV results in onset of a B lymphoid leukemia/lymphoma. Whereas $100 \%$ of TYK2-/- animals died of leukemia/lymphoma within 10 weeks, $30 \%$ of the heterozygous littermates survived the oncogenic challenge for more than 6 months; a log rank test confirmed the significance of this difference $(P=0.0036)$ (Figure $1 \mathrm{~A})$. The phenotype of the disease in TYK2 ${ }^{+/-}$and TYK2 $2^{-/-}$mice was comparable: all diseased mice had elevated white blood cell counts consisting of CD $19^{+} \mathrm{CD} 43^{+}$cells, which also infiltrated spleen and liver (Figure 1, B-D). It is worth pointing out that the white blood cell counts in TYK2 $2^{-/-}$mice were significantly higher than those found in the diseased control animals $\left(37 \times 10^{3}\right.$ cells per microliter versus $19 \times 10^{3}$ cells per microliter in TYK2-/- and control animals, respectively; $P=0.005)$. In contrast, the enlargement of spleen, liver, and lymph nodes was comparable in $\mathrm{TYK} 2^{+-}$and TYK2 $2^{--}$diseased mice. Histological sections revealed a comparable infiltration of leukemic cells in the liver and a pronounced extramedullary hematopoiesis in the spleen in both TYK2 ${ }^{+/-}$and TYK2-/- animals (Figure 1D).

Equal numbers of pro-B cells in TYK2-/- mice. To investigate whether the altered incidence of disease is caused by variable numbers of susceptible target cells for the Abelson oncogene in vivo, we analyzed the numbers of pro-B cells in the bone marrow of TYK2-/mice. Bone marrow of 10 age-matched TYK2 knockout mice and 10 controls was analyzed by FACS. The numbers of CD $19+C D 43^{+}$ B cell precursors were comparable in both mouse strains $(n=18$; $19.3 \% \pm 6.5 \%$ and $21.5 \% \pm 4.3 \%$ in WT and TYK $2^{-/-}$mice, respectively). One representative example is depicted in Figure 2A. Total cellularity of the bone marrow varied depending on the age of the mice but was comparable in age-matched mice of either genotype. Alternatively, a higher proliferation rate of TYK2 $2^{-/}$pro-B cells might increase viral infection rates. To address this issue, pro-B cells were enriched to $85 \%$ purity by cultivation of bone marrow of TYK2 $2^{+-}$and TYK2-/- mice for 7 days in IL-7. The cells thus enriched were subjected to a $\left[{ }^{3} \mathrm{H}\right]$ thymidine incorporation assay (Figure 2B). No differences were observed in the proliferative response of $\mathrm{TYK}^{+/-}$and $\mathrm{TYK} 2^{-/-} \mathrm{B}$ lymphoid precursor cells. Hence, we can rule out that the higher incidence of leukemia in TYK $2^{-/-}$mice is related to an elevated number or an increased growth rate of pro-B cells.
In vitro transformation assays and tumor cell injections in nude mice. The increased incidence, decreased latency, and increased aggressiveness of Abelson-induced tumors in TYK2 $2^{-/}$mice may also be explained by an increased transformation rate in TYK2 $2^{-/}$B lymphoid cells compared with WT cells. To explore this possibility, in vitro transformation assays were performed. Loss of TYK2 did not affect the number of A-MuLV-transformed growth factor-independent colonies. If there was a difference at all there were even fewer colonies derived from TYK2 $2^{-/-}$mice (Figure $2 \mathrm{C}$ ). The colonies did not differ in size, which would have indicated that the cells had an accelerated growth rate. Immunophenotyping of the cells within the colonies identified them as $\mathrm{CD} 19^{+} \mathrm{B} 220^{+}$B lineage cells (data not shown). Similarly, IL-7-dependent colony formation of the infected cells was unaltered in TYK2 $-/-$ mice, which indicates that growth factor-dependent proliferation of the transformed cells was also unchanged (Figure 2D).

In addition, the lack of TYK2 did not alter the activation status of the downstream STAT transcription factors in the leukemic cells. We found a consistent activation of STAT1, STAT3, and STAT5 in primary tumor samples (Figure 2E and data not shown).

A cell-autonomous effect of TYK2 deficiency is also unlikely in view of the fact that equal numbers of cell lines with comparable growth characteristics grew out of transformed bone marrow preparations (data not shown). Additional proof was obtained by injection of TYK2 $2^{-/-}$and $\mathrm{TYK}^{+/-}$transformed cell lines into nude mice, either s.c. or into the tail vein to mimic the leukemic situation. In this experimental setting the tumor cells are confronted with the same conditions, namely a reduced tumor surveillance system mainly consisting of NK cells from the host nude mice.

Three individually in vitro-derived TYK2 ${ }^{+/-}$and TYK $2^{-/-}$cell lines were injected s.c. This resulted in tumor formation with equal latency and tumor weight in all nude mice ( $n=12$ for each genotype; weight, $0.48 \pm 0.11 \mathrm{~g}$ and $0.43 \pm 0.046 \mathrm{~g}$ for TYK2 $2^{+--}$and TYK $2^{-/-}$ mice, respectively). A-MuLV-infected IFN- $\gamma^{-/-}$cell lines and their corresponding WT cell lines were used as control for the experimental setting. As previously described (16) v-abl-transformed IFN $-\gamma^{-/-}$cells gave rise to significantly larger tumors $(2.1 \pm 0.47 \mathrm{~g}$ and $0.59 \pm 0.31 \mathrm{~g}$ for IFN $-\gamma^{-/-}$cells and IFN- $\gamma^{+/+}$cells, respectively), underlining that the role of TYK 2 and IFN- $\gamma$ in Abelson-induced tumor formation cannot be superimposed. 
A

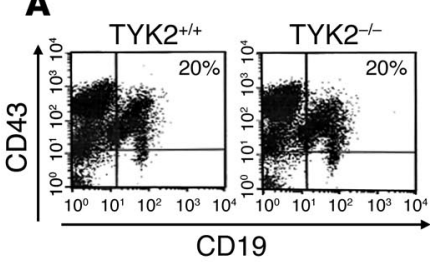

B
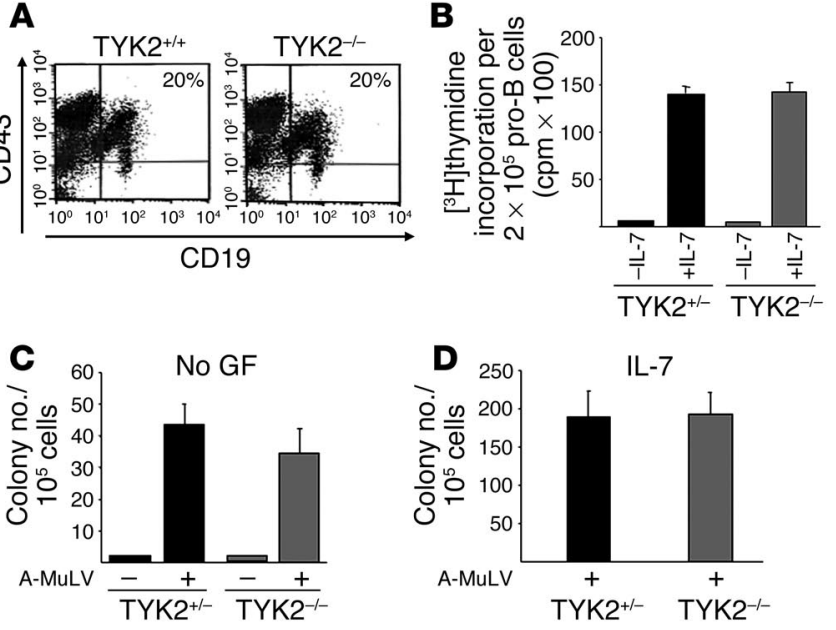

E Antibody - S1 S3 - S1 S3

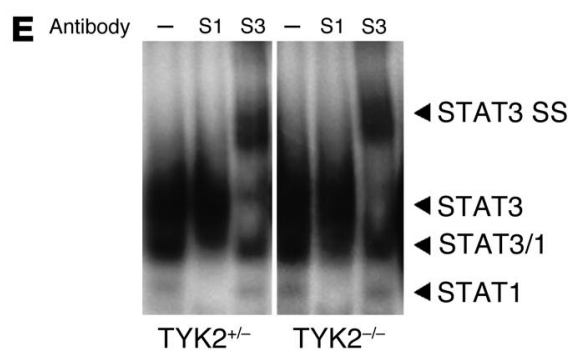

Similarly, injection of tumor cells via the tail vein led to a leukemic phenotype in nude mice within 4 weeks. The latency of the disease was identical irrespective of whether TYK2 $2^{-/-}$or TYK2 $2^{+/-}$ cells had been injected (Figure 3A). The spleen weight (Figure $3 \mathrm{~B})$ and the infiltration of leukemic cells in the bone marrow (Figure 3C) were comparable. White blood cell counts were elevated to the same range, and the leukemic cells did not differ morphologically or in their surface markers $\left(\mathrm{CD} 43^{+} \mathrm{CD} 19^{+}\right)$ (data not shown). Hence, transformed TYK2-/- and WT cells have a virtually identical malignant phenotype when injected into nude mice.

TYK2 is essential for the evolutionary sculpting of transformed B lymphoid cells. Taken together, these experiments ruled out that a cell-autonomous effect of TYK2 deficiency is responsible for the enhanced susceptibility of TYK2-/- mice to the leukemic challenge. We next asked whether tumor surveillance was impaired in TYK2-/mice. A reduced tumor surveillance results in an impaired evolutionary pressure on the tumor which may be analyzed by experiments using transplantation into syngeneic immunocompetent hosts (19). Tumor cells that have not been under the pressure of a functional immune system will be recognized and rejected more easily by an immunocompetent host.

\section{Figure 3}

Tumor formation in nude mice. (A) Kaplan-Meier plot of nude mice after tail vein injection with $10^{6}$ transformed cells. No difference in the course of the disease was detected, irrespective of whether TYK2 ${ }^{+/-}$or TYK2 $^{-/-}$transformed cells were injected ( $n=8$ mice for each genotype). (B) Mean spleen weights of nude mice injected with TYK2 $2^{+-}$or $\mathrm{TYK}^{-/-}$transformed cells. Data show means $\pm \mathrm{SE}(n=8$ for each genotype). (C) Infiltration of CD19+ cells in bone marrow was determined by FACS. Data show means \pm SE ( $n=8$ for each genotype).

\section{Figure 2}

In vitro characterization of $\mathrm{B}$ lymphoid transformation. (A) CD19+CD43+ $\mathrm{B}$ lymphoid precursor cells of TYK2 ${ }^{+/+}$and TYK2 $2^{-/-}$bone marrow were identified by FACS analysis. One typical example is depicted ( $n=10$ for each genotype). (B) Bone marrow of TYK2 ${ }^{+/}$and TYK2 ${ }^{-/-}$mice was kept in IL-7-containing medium for 7 days and subsequently applied to a $\left[{ }^{3} \mathrm{H}\right]$ thymidine incorporation assay. ( $\mathbf{C}$ and D) Single-cell suspensions derived from bone marrow were infected with A-MuLV and subsequently cloned in growth factor-free (no GF) (C) or IL-7-containing methylcellulose (D). Mock-infected cell preparations are included as controls (C). No significant differences were observed. The initial number of B cells in each individual bone marrow preparation was determined by FACS. Results show the average numbers \pm SD of colonies obtained $\left(n=6\right.$ for TYK2 ${ }^{+-}$and $n=6$ for $\mathrm{TYK}^{-/-}$bone marrow). (E) STAT activation pattern was analyzed by electrophoretic mobility shift assay and supershift analysis in 3 individual $\mathrm{TYK}^{+/-}$and $\mathrm{TYK}^{-/-}$primary tumor samples. One representative example of each genotype is shown. Antibodies directed against STAT1 (S1) and STAT3 (S3) reveal an activation of STAT1 and of STAT3 as indicated by supershifts (SS).

Transplantation experiments were performed, and the results are listed in Table 1. Each tumor-derived cell suspension was injected into 2 recipient mice. The injected cell lines did not differ in their proliferative capacity or viability. Cell viability was routinely checked upon injection of the cells. Cell proliferation was analyzed by $\left[{ }^{3} \mathrm{H}\right]$ thymidine incorporation in 4 individual $\mathrm{TYK}^{+/-}$and $\mathrm{TYK} 2^{-/-}$tumor-derived cell preparations at the time point of injection and was found to be comparable $\left(179 \times 10^{3} \pm\right.$ $47 \times 10^{3} \mathrm{cpm}$ versus $191 \times 10^{3} \pm 87 \times 10^{3} \mathrm{cpm}$ for $\mathrm{TYK} 2^{+/-}$versus TYK2 $2^{-/-}$tumor cells, respectively). Within 2 weeks, 12 of 16 mice (75\%) developed tumors if TYK2 $2^{+-}$tumor cells had been injected. Conversely, only 5 of 18 mice (28\%) developed tumors if TYK2-/cells had been injected. In some of the mice that had received TYK2-/- tumor cells we observed a transient nodule, which disappeared within 2 weeks. This transient swelling is most likely due to an inflammation process at the site of injection. The difference in tumor incidence meets the criteria of statistical significance $(P=0.006)$, as does the difference in tumor weight $(0.33 \pm 0.06 \mathrm{~g}$ versus $0.09 \pm 0.04 \mathrm{~g} ; P=0.004)$.

Tumor surveillance components in TYK2 $2^{-1-}$ mice. The key players in tumor surveillance are CTLs, NK cells, and NK T cells (NKT cells). We first investigated whether TYK2 $2^{-/-}$mice differed in their numbers of CTLs, NK cells, and NKT cells. FACS analysis of lymph nodes of WT and TYK2 $2^{-/-}$mice did not reveal any differences in the

A
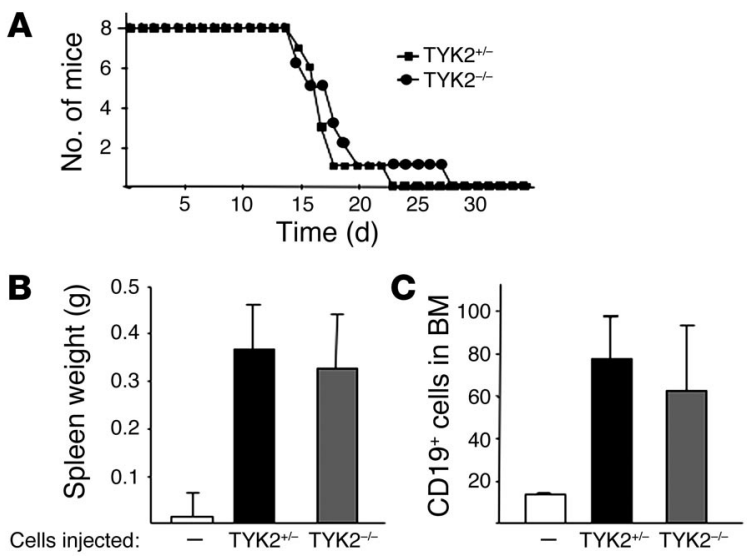
Table 1

Tumor transplantation into WT mice

$\begin{array}{cc}\text { +/- Tumor weight }(\mathbf{g}) & \text {-/- Tumor weight }(\mathbf{g}) \\ 0.4 & 0 \\ 0.42 & 0.24 \\ 0 & 0 \\ 0 & 0 \\ 0.81 & 0.23 \\ 0.2 & 0 \\ 0.29 & 0 \\ 0.53 & 0 \\ 0 & 0 \\ 0 & 0.2 \\ 0.78 & 0 \\ 0.17 & 0 \\ 0.42 & 0 \\ 0.43 & 0 \\ 0.39 & 0 \\ 0.29 & 0 \\ & 0.51 \\ & 0.5\end{array}$

Tumors were induced in newborn TYK2 $2^{+/-}$and TYK2 $2^{-/-}$mice by injection of A-MuLV. The tumors were excised, and $2 \times 10^{6}$ tumor-derived cells were injected s.c. into WT C57BL/6 mice. The weights of these secondary tumors are listed.

composition of lymph nodes, and $\mathrm{CD} 4^{+} \mathrm{T}$ cells, $\mathrm{CD} 8^{+} \mathrm{T}$ cells, $\mathrm{NKT}$ cells, and NK cells were present in equal numbers (Figure 4A). The size of the lymph nodes and of the thymus did not differ between age-matched mice of different genotypes, which indicates that TYK2 did not affect cellularity. Accordingly, total cell counts were comparable (data not shown). The impact of CTLs and NK/NKT cells in tumor surveillance strongly depends on the origin of the tumor cell and varies among individual tumor types. Tumor cells that are primarily eliminated by CTLs downregulate MHC class I expression to evade recognition. Primary tumor-derived cells ( $n=4$ of each genotype) were analyzed by FACS. Irrespective of the genotype, all tumors expressed high levels of MHC class I molecules, which strongly argues against a major involvement of CTL cells (Figure 4B). In addition, we failed to induce a CTL response by immunizing C57BL/ 6 mice with in vitro-transformed A-MuLV cells, whereas immunization with the adenocarcinoma cell line 38.2 induced CTL-dependent killing of target cells (data not shown). We therefore focused our attention on NK/NKT cells and analyzed

\section{Figure 4}

Analysis of lymph nodes and primary lymphoma. (A) Lymph nodes of $\mathrm{TYK}^{+/+}$, $\mathrm{TYK}^{+/-}$, and TYK2 ${ }^{-/-}$mice $(n=4$ each) were excised and analyzed for the composition of B, T, NK, and NKT cells by FACS. One representative example of each genotype is depicted. Numbers of $\mathrm{CD} 4^{+} \mathrm{T}$ cells and $\mathrm{CD} 8^{+} \mathrm{T}$ cells in the lymph nodes are shown in the upper panels and numbers of NK cells (NK1.1+CD3-), NKT cells (NK1.1+CD3+), and CTLs (NK1.1-CD3+) in the lower panels. (B) MHC class I expression of TYK2 ${ }^{+/-}$and TYK2 ${ }^{-/-}$tumor-derived cell lines was analyzed by FACS ( $n=4$ for each genotype). One representative example is depicted; no differences were observed in any of the samples investigated. (C) Lymphomas of TYK2 ${ }^{+/-}$and TYK2 $2^{-/-}$mice were excised and analyzed for infiltrated NK cells (left panel) and NKT cells (right panel) by FACS ( $n=7$ individual TYK2 ${ }^{-1-}$ mice and $n=10$ individual TYK2 ${ }^{+-}$mice).

their infiltration into primary lymphomas. Indeed, we found that NK/NKT cells infiltrated the evolving lymphomas (Figure 4C).

In vitro characterization of TYK $2^{--}-N K / N K T$ cells. In order to closely investigate the properties of TYK2-/- NK/NKT cells, we amplified them in vitro. Growth kinetics of TYK2 $-/$ NK cells did not differ from those of their TYK2 $2^{++}$counterparts. In either instance, the selective medium allowed for the isolation of pure NK1.1 $1^{+}$cell populations (Figure 5, A and B). The percentages of NKT cells $\left(\mathrm{NK} 1.1^{+} \mathrm{CD}^{+}\right)$in those cultures were identical $(15.0 \% \pm 3.5 \%$ versus $15.2 \% \pm 4.6 \%$ in TYK2 $2^{+/+}$and TYK2 $2^{--}$cell cultures, respectively). Signaling leading to the activation of the MAPK isoforms ERK1 and ERK2 was unimpaired when NK/NKT cells were challenged with IL-2, as was the activation of STAT5, which is thought to be essential for the growth-stimulatory effects of IL-2 (data not shown).

However, TYK2 $2^{--}$NK/NKT cells produced less IFN- $\gamma$ under basal nonstimulated conditions (48 hours of incubation in the absence of IL-2) as well as upon stimulation with IL-12, as shown by ELISA. The differences in basal IFN- $\gamma$ and IL-12-induced IFN- $\gamma$ production were statistically significant $(P \leq 0.05)$ (Figure 5C). IFN- $\gamma$ exerts an inhibitory and proapoptotic effect on tumor cells and is considered a key player in tumor surveillance (32). When we analyzed primary tumors for the presence of IFN- $\gamma$, we found vari-

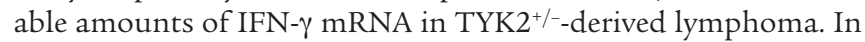
contrast, IFN- $\gamma$ mRNA was consistently undetectable in TYK2 $2^{-/-}$ derived lymphoma (Figure 5D). The lack of TYK2 did not impede the responsiveness of Abelson-transformed pro-B cells to IFN- $\gamma$; growth inhibition was induced to the same extent and with comparable $50 \%$ effective concentration $\left(\mathrm{EC}_{50}\right)$ values $(0.3 \mathrm{ng} / \mathrm{ml})$ in tumor-derived cells in vitro (data not shown). IL-12 may participate in tumor surveillance by activating NK cells. However, although the primary tumors expressed the p35 subunit of IL-12, p40 was not expressed, irrespective of the genotype (data not shown).

The second major NK/NKT cell-mediated mechanism of tumor defense, cytolytic ability, was also decreased (Figure 5E) when analyzed in a cytotoxicity assay using YAC- 1 cells as target cells.
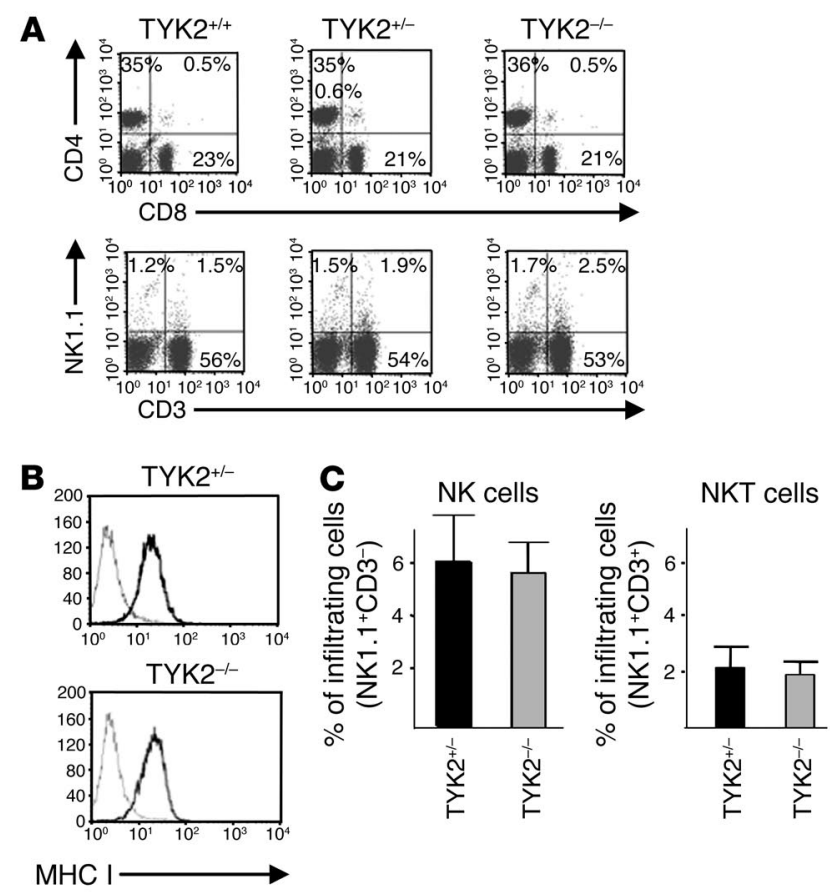

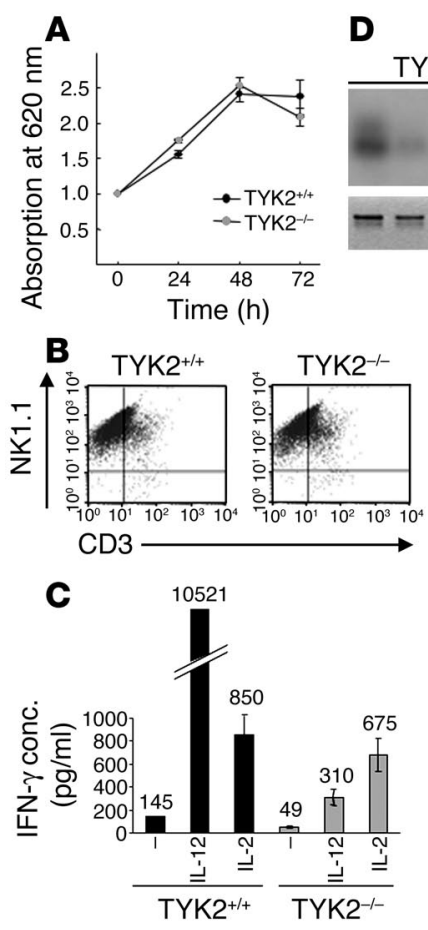

\section{Figure 5}

In vitro characterization of TYK2+/- and TYK2 ${ }^{-1-}$ NK/NKT cells. (A) An MTT assay did not reveal any differences in growth characteristics of TYK2 $2^{+/+}$-derived and TYK2 $2^{-/-}$-derived NK/NKT cells. Each data point represents mean \pm SD from 5 individual wells. One representative experiment is depicted $(n=3)$. (B) Similarly, FACS analysis of the in vitro-derived cells showed a comparable composition of NK $\left(\mathrm{NK} 1.1^{+} \mathrm{CD}^{-}\right)$and NKT $\left(\mathrm{NK} 1.1^{+} \mathrm{CD}^{+}\right)$cells, with an estimate of $20 \%$ NKT cells. One representative experiment is depicted $(n=6$ for each genotype). (C) Production of IFN- $\gamma$ in NK/NKT cells after expansion with IL-2. NK/NKT cells were either left untreated or incubated with IL-12 or IL-2 for 48 hours. Culture supernatants were analyzed for IFN- $\gamma$ production by ELISA. One representative experiment is shown $(n=4)$. (D) RT-PCR and subsequent oligohybridization for IFN- $\gamma$ from TYK2+/derived and TYK2 ${ }^{-l-}$-derived tumors. Representative examples of the analysis are shown in the upper panel. Activated (IL-2 and anti-CD3) primary T lymphocytes were used as positive control (Co). An RT-PCR reaction for $\beta$-actin was performed to control for the prepared cDNA. IFN- $\gamma$ was only detected in tumors derived from TYK2 $2^{+-}$animals. (E) Cytotoxicity assay using TYK2 ${ }^{+/+}$and $\mathrm{TYK}^{-/-} \mathrm{NK} / \mathrm{NKT}$ cells as effector and YAC-1 cells as target cells. One representative experiment is shown $(n=4)$. TYK2 ${ }^{-/-}$NK/NKT cells were consistently less efficient in lysing YAC-1 cells. E/T, effector/target; conc., concentration.
TYK2 $2^{-1-}$ NK/NKT cells have an impaired ability to lyse their homologous tumor-derived target cells. YAC-1 cells are recognized by NK/NKT cells because of their low expression of MHC class I molecules. However, the relevant issue is whether TYK2-/- NK/NKT cells are less efficient in killing primary tumor-derived target cells. We used TYK2--- and WT NK/NKT cells as effectors and tumor-derived cells of both genotypes as targets. Each possible combination was tested, and representative findings are depicted in Figure 6, A and B. Although the maximal cytotoxicity varied among the individual assays, we consistently found a reduced function of TYK2-/- NK/NKT cells. This impairment was independent of the genotype of the target cells and met the criteria of statistical significance $(P=0.03)$ (Figure 6 , A and $B$ ).

RAG2-/-TYK2-/- mice confirm the key role of $N K$ cells in rejection of Abelson-induced tumors. RAG2 ${ }^{-/}$mice lack functional B and $\mathrm{T}$ lymphocytes as well as NKT cells (27). Hence, the tumor surveillance system of RAG2 $2^{-/}$mice entirely depends on the recognition and elimination of transformed cells by NK cells. We intercrossed RAG2-/- mice with TYK2 ${ }^{-/-}$mice to generate double-knockout animals. Abelson infection of newborn RAG2 ${ }^{-/-T Y K 2-/-}$ animals resulted in a significantly $(P=0.0027)$ earlier disease onset than Abelson infection of

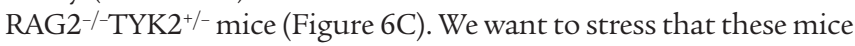
are on a mixed genetic background. Since the genetic background alters the susceptibility to the Abelson oncogene, we included control mice with an identical mixed genetic background (RAG2 $2^{+-}$ $\mathrm{TYK}^{+/-}$and $\mathrm{RAG}^{+/-} \mathrm{TYK}^{-/-}$animals) in the experiment (data not shown). Irrespective of whether NK cells alone battled the evolving tumor (RAG2 $2^{-/-}$) or whether the entire immune system was available for tumor surveillance (RAG2 $2^{+-}$), the difference in disease latency between $\mathrm{TYK}^{+/-}$and TYK2 $2^{-/-}$mice remained constant. These experiments define NK cells as major players in the fight against Abelsoninduced B lymphoid leukemia/lymphoma and pose TYK2 as a key molecule for the tumor surveillance of this disease.

TYK2 deficiency enhances tumor susceptibility to other lymphoid malignancies. In order to investigate whether the increased susceptibility of TYK2 $2^{-/-}$animals is a general feature in the tumor surveillance of lymphoid malignancies, additional tumor models were tested. First, we intercrossed TEL-JAK2 transgenic mice and TYK2-/- animals. The TEL-JAK2 fusion results from the $\mathrm{t}(9 ; 12)$ chromosomal translocation found in human lymphoid and myeloid leukemia $(14,32)$. If the TEL-JAK2 fusion protein is expressed under the control of a lymphoid enhancer in transgenic mice, $\mathrm{T}$ lymphoid leukemia/lymphoma arises at the age of 10-20 weeks (15). Leukemia onset was significantly accelerated on a TYK2-/- background $(P=0.0273)$ (Figure 7A). Apart from the differences in latency, the phenotype of the disease was comparable: TYK2 $2^{-/-}$and TYK2 ${ }^{+/-}$TEL-JAK2 transgenic animals developed a $\mathrm{CD}^{+} \mathrm{T}$ lymphoid leukemia with thymoma.

In addition, the murine $\mathrm{T}$ cell lymphoma line EL-4 (33-35) was injected s.c. into age-matched TYK $2^{+/+}$and TYK $2^{-/-}$mice. The experiment was terminated 15 days thereafter by analysis of tumor masses. TYK2 deficiency allowed for the development of tumors that were significantly larger in mass than those in the WT controls $(n=6$ for each genotype; weight, $0.13 \pm 0.079 \mathrm{~g}$ and $0.476 \pm 0.076 \mathrm{~g}$ for TYK2 ${ }^{+/+}$and TYK2 ${ }^{-/-}$mice, respectively; $P=0.0099$ ) (Figure $7 \mathrm{~B}$ ).

We did not observe spontaneous lymphoma formation in a cohort of $47 \mathrm{TYK} 2^{-/-}$mice that were observed for a period of over 12 months. A single mouse developed an epithelial carcinoma, a frequency of spontaneous tumor formation that is within the normal range.

\section{Discussion}

Given the essential role of the JAK-STAT pathway in cell growth and survival, it is not surprising that inappropriate activation of its signaling components has been observed in solid tumors as well as in leukemia $(4,36)$. JAKs may drive transformation upon constitutive activation; this is best exemplified by the TEL-JAK2 fusion protein $(15,16)$. Moreover, JAK2 has been implicated as a mediator of bcr/ abl-induced transformation, in which JAK2 regulates the expression of c-myc (37). Recent evidence, however, indicated a role of JAK1 as 

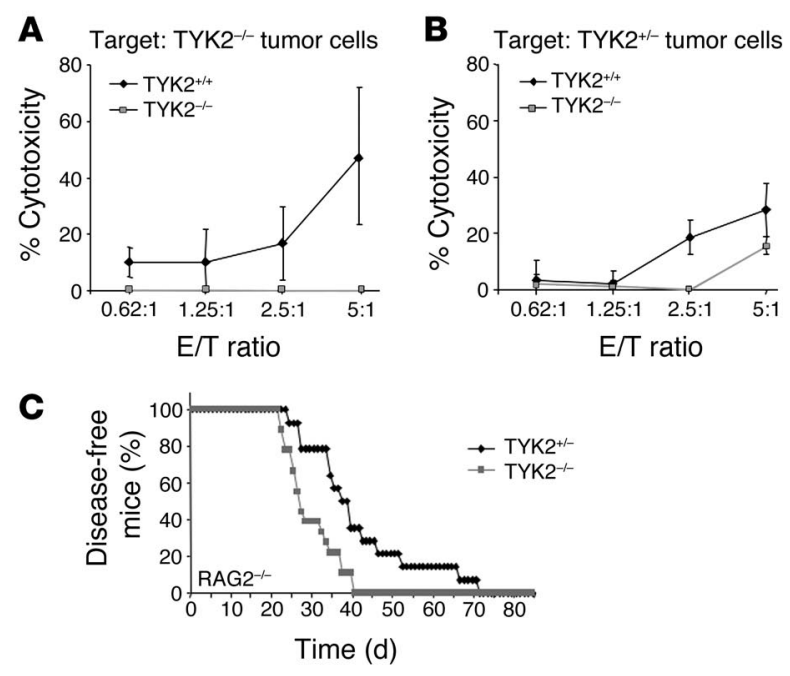

a tumor suppressor for Abelson-induced B cell lymphoma (16). In view of these interesting findings, we investigated the role of the closely related Janus kinase TYK2 in hematological malignancies.

The viability of TYK2 $2^{-/-}$mice enabled us to use an experimental setup that allowed investigation of both cell-autonomous transformation processes and the functionality of the tumor surveillance system. The inflicted disease develops slowly, requires additional epigenetic changes, and mimics tumor development in humans. The prolonged development of disease enables the immune system to take corrective action and efficiently reject the tumor. This is exemplified by the fact that only $70 \%$ of WT animals are affected. In contrast, $100 \%$ of TYK2-/- animals succumb to leukemia/lymphoma. In this regard this experimental system is superior to bone marrow transplantation studies that provoke disease much faster because of a higher initial tumor burden.

A slowly evolving leukemia is also induced by the TEL-JAK2 transgene in mice. Again, TEL-JAK2/TYK2-/- transgenic mice succumbed to disease significantly faster than their TEL-JAK2/ TYK2 ${ }^{+/-}$littermates. This observation extends the importance of TYK2 for lymphoid tumor surveillance to nonvirally induced tumors of B and $\mathrm{T}$ lymphoid origin. In addition, tumor formation by s.c. injection of low numbers of the lymphoma cell line EL-4 was significantly accelerated in TYK2 $2^{-/}$animals, which underlines the general importance of TYK2 for lymphoid tumor rejection.

Our findings rule out that TYK2 is involved in the initial process of transformation by A-MuLV. First, we failed to detect any differences in the assays in vitro. Growth factor-independent colony formation in response to the Abelson oncogene was unaltered, as was the outgrowth of immortalized cell lines. Finally, WT and TYK2-/Abelson-transformed cells result in the same disease latency, severity, and phenotype upon injection into nude mice, irrespective of whether the cells were injected s.c. or via the tail vein.

Tumor development is an interplay and a permanent battle between the evolving tumor and the surrounding immune system (17). NK cells are considered the first line of defense against hematopoietic malignancies $(38,39)$. NK cells - in conjunction with NKT cells - kill tumor cells of the hematopoietic lineage without additional stimulus. In fact, NK cells have been shown to be highly efficient against Abelson-transformed cells $(40,41)$.

Our data strengthen this concept. TYK2 $2^{-/-}$NK cells fail to lyse tumor target cells efficiently. This defect is paralleled by an

\section{Figure 6}

Effect of TYK2 ${ }^{-/}$NK/NKT cells on tumor target cells. (A and B) Cytotoxicity assay using in vitro-expanded NK/NKT cells of WT (diamonds) and TYK2 ${ }^{--}$animals (squares) as effector cells and the $\mathrm{TYK}^{-1-}$ tumor-derived cell line (A) or the TYK2 ${ }^{+/-}$cell line (B) as target cells. Irrespective of the target cells, TYK2 ${ }^{-1-}$-derived NK/NKT cells had a reduced cytolytic ability. Each assay combination was tested at least 3 times. One representative experiment is depicted. (C) KaplanMeier plot of TYK2 ${ }^{+-}$and TYK2 $2^{--}$mice on a RAG2 ${ }^{-1-}$ background after injection of a replication-incompetent A-MuLV retrovirus ( $n=14$ for TYK2 $2^{+-}$and $n=18$ for TYK2 $^{---}$). RAG2 $2^{--}$-TYK2 $2^{--}$animals succumbed significantly earlier to Abelson-induced disease than their RAG2 ${ }^{-/-}$ TYK2 $^{+-}$littermates as determined by a log rank test $(P=0.0027)$. increased incidence of leukemia/lymphoma formation in TYK2-/animals. The lack of TYK2 does not inhibit invasion of NK/NKT cells into the evolving lymphoma, since the numbers of NK and NKT cells in TYK2-/- lymphoma were comparable to those in WT controls. Infiltration of immune cells into tumors is controlled by chemokine receptors, which activate JAK-STAT signaling cascades $(42,43)$. Accordingly, our observations exclude a role for TYK2 in tumor invasion.

Besides NK/NKT cells, CTLs are thought to play an important role in tumor rejection. CTLs recognize tumor targets via MHC class I-presented antigen. The involvement of CTLs in tumor surveillance provokes the downregulation of MHC class I on the tumor cell surface $(44,45)$. This downregulation is a very common feature of tumors evolving under the selective pressure of CTLs. We failed to detect any alterations of MHC class I expression. Hence, it is highly unlikely that CTL-mediated cytotoxicity has a prominent impact in our tumor system. Moreover, immunization of C $57 \mathrm{BL} / 6$ mice with in vitro-transformed A-MuLV cells failed to provoke a CTL response in vivo, whereas immunization with an adenocarcinoma cell line resulted in a pronounced CTL-dependent killing. Most importantly, TYK2-/-
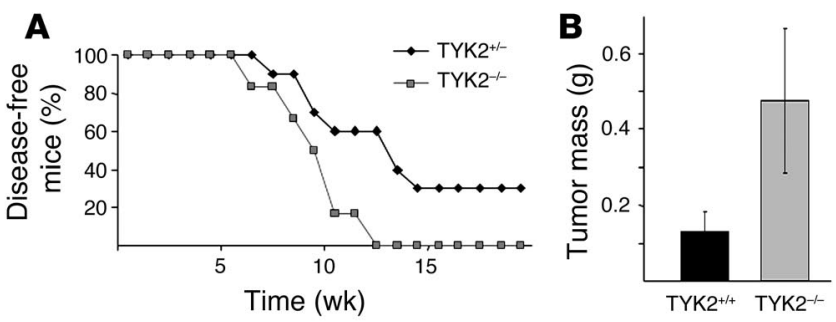

\section{Figure 7}

Effect of TYK2 deficiency on tumor surveillance in other lymphoid malignancies. (A) Kaplan-Meier plot of TEL-JAK2 transgenic TYK2 ${ }^{+/-}$ and TEL-JAK2 transgenic TYK2 $2^{-/-}$mice $\left(n=10\right.$ for TEL-JAK2/TYK2 $2^{+-}$ and $n=6$ for TEL-JAK2/TYK2 ${ }^{--}$). TEL-JAK2 transgenic TYK2 ${ }^{-/-}$animals developed disease significantly earlier when compared with their TEL-JAK2 transgenic TYK2 ${ }^{+/-}$littermate controls as determined by a log rank test $(P=0.0273)$. (B) Mean tumor weights of TYK2 $2^{+/+}$and TYK2 ${ }^{-1-}$ animals injected with $10^{5}$ EL-4 cells. Data show means $\pm \mathrm{SE}$ ( $n=6$ for each genotype; weight, $0.13 \pm 0.079 \mathrm{~g}$ and $0.476 \pm 0.076 \mathrm{~g}$ for TYK2 ${ }^{+/+}$and $\mathrm{TYK}^{-/-}$mice, respectively; $P=0.0099$ ). 
mice showed a comparable reduction in leukemia/lymphoma latency when compared with $\mathrm{TYK} 2^{+/-}$animals on a RAG2-/- background. In this experimental setting, NK cells are the only operators of tumor surveillance. Control experiments on TYK2 ${ }^{-/-}$and $\mathrm{TYK} 2^{+/-}$mice on a RAG2 ${ }^{+/-}$background (using the sibling mice of the backcrosses) did not further enhance the differences in disease latency despite the availability of CTLs and NKT cells. A prominent role of CTLs in tumor surveillance would indeed significantly alter disease onset, since TYK2-/- CTLs have a drastically reduced cytotoxic capacity (C. Schellack and D. Stoiber, unpublished observations).

Similarly, tumor surveillance of TEL-JAK2-induced leukemia depends on NK cells. Intercrossing TEL-JAK2 transgenic animals with TCR $\alpha^{-/-}$mice does not shift disease phenotype or latency $(\mathrm{N}$. dos Santos and J. Ghysdael, personal communication). The situation for EL-4-induced lymphoma is less clear cut, because NK cells as well as CTLs have been reported to lyse EL-4 cells (33-35).

IFN- $\gamma$ is thought to be the key component in tumor surveillance. We detected IFN- $\gamma$ mRNA in about $60-70 \%$ of WT-derived lymphoma but failed to see IFN- $\gamma$ in any of the TYK2-/- lymphomas. Although we are currently unable to define the origin of IFN- $\gamma$, we speculate that the main source of IFN- $\gamma$ is the NK/NKT cell itself. TYK2 ${ }^{-/-}$NK/NKT cells have been described to produce less IFN- $\gamma$ (46), a finding that we confirmed (Figure 5C). However, the role of TYK2 in tumor surveillance cannot be simply accounted for by a lack of IFN- $\gamma$-dependent signaling. IFN- $\gamma$ and TYK2 deficiency cannot be superimposed. A-MuLV-transformed IFN- $\gamma^{-/}$cells show an increased tumor formation upon subcutaneous injection in nu/nu mice (16); this effect is not observed upon injection of TYK2 $2^{-/-}$cells. Similarly, the development of leukemia is accelerated upon tail vein injection of A-MuLV-transformed IFN- $\gamma^{-/-}$cells but not upon injection of TYK2-/- cells into nu/nu animals (data not shown and Figure 3).

Although IL-12 is known to stimulate NK cells and thereby may contribute to tumor surveillance in some tumors, it is irrelevant to our experimental system $(47,48)$. We failed to detect the p40 subunit of IL-12 in primary lymphomas, whereas we readily showed IFN- $\gamma$ mRNA in primary lymphoma. This observation is in line with a recent report: When bred on a C57BL/ 6 background, IFN- $\gamma^{-/-}$animals developed lymphoma, whereas no spontaneous malignancies evolved in IL-12-/- mice (21).

Our data support the recent concept that lymphoid cells and IFN- $\gamma$ may be overlapping forces in tumor surveillance. A central tenet of this concept is the postulation that any evolving tumor is sculpted by the immune system $(17,19)$. Primary tumors derived from $\mathrm{TYK} 2^{-/-}$animals were rejected to a significantly higher degree than primary tumors derived from heterozygous controls when transplanted in WT mice of identical genetic origin. Evolutionary pressure of the immune system was absent in TYK2-/- animals; the tumor cells had not acquired the ability to circumvent the selective pressure by the WT host.

It is tempting to speculate that TYK2 deficiency in people is clinically silent (unless virus load is excessive) but predisposes to tumor formation. We propose that patients with leukemia or other hematopoietic malignancies represent a population in which TYK2 deficiency is enriched. Thus, the presence or absence of TYK2 in patients needs to be addressed in the future. Absence of TYK2 would also predict a reduced responsiveness to type I IFNs, which are used, for example, for the treatment of chronic myeloid leukemia. Consequently, these patients would have to be treated by alternative means. Therefore, TYK2 deficiency may be relevant to the prognosis of patients as well as to their proper treatment.

\section{Methods}

Cells and virus preparation. Transformed fetal liver cells, bone marrow cells, and tumor-derived cell lines were maintained in RPMI medium containing $10 \% \mathrm{FCS}, 100 \mathrm{U} / \mathrm{ml}$ penicillin/streptomycin, and $5 \mu \mathrm{M}$ $\beta$-mercaptoethanol. A010 cells that produce an ecotropic replicationdeficient form of the Abelson virus were maintained in DMEM containing $10 \% \mathrm{FCS}$ and $100 \mathrm{U} / \mathrm{ml}$ penicillin/streptomycin. Viral supernatant was prepared as previously described (49).

Mice and infection of neonatal mice with A-MuLV. TYK2 $2^{-/-}$mice were described previously (25) and were backcrossed to C57BL/ 6 for 8 generations. Nude mice were purchased from the Institut für Labortierkunde und Genetik (Himberg, Austria).

For the infection of newborn animals, TYK2 ${ }^{+/-}$were bred with TYK2 $2^{-/-}$animals. Hence, every litter contained TYK2 $2^{+-}$and TYK2 $2^{-/-}$animals to ensure appropriate controls. We initially started the in vivo transformation experiments by intercrossing $\mathrm{TYK}_{2}^{+/-}$and $\mathrm{TYK}^{+/-}$mice and analyzed leukemia/ lymphoma formation in all 3 genotypes: $\mathrm{TYK}^{+/+}, \mathrm{TYK} 2^{+/-}$, and $\mathrm{TYK} 2^{-/-}$. We have not observed any differences between $\mathrm{TYK}^{+/+}$and $\mathrm{TYK} 2^{+/-}$mice. In all experiments where age-matched littermate controls were necessary (transformation assays), $\mathrm{TYK}^{+/-}$and TYK2 $2^{-/-}$mice were intercrossed to optimize controls. For all other purposes, TYK2 ${ }^{+/-}$were replaced with $\mathrm{TYK} 2^{+/+}$mice that do not require permanent genotyping of the mouse offspring.

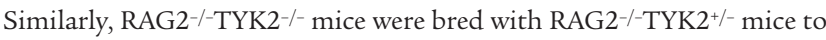
ensure appropriate controls within 1 litter (50). Newborn mice were injected i.p. with $50 \mu \mathrm{l}$ of replication-incompetent ecotropic retrovirus encoding for v-abl. The mice were checked daily for onset of diseases, and sick mice were sacrificed and analyzed (49). All animal experiments were approved by the Tierversuchskommission and were carried out in accordance with protocols approved by Austrian law.

Infection offetal liver and bone marrow and in vitro transformation assays. Fetal liver cells were prepared and single-cell suspensions were infected as previously described (51). Similarly, single-cell suspensions of bone marrow of tibiae and femora of 6 -week-old mice were infected. After 24 hours, the cells were washed several times and plated in methylcellulose at a density of $3 \times 10^{5}$ cells per milliliter in $35-\mathrm{mm}$ dishes. An aliquot was plated in methylcellulose enriched with $10 \mathrm{ng} / \mathrm{ml}$ IL-7 (Stem Cell Technologies Inc.). After 7 days, the cloning efficiency was determined by counting of the foci using light microscopy. Some colonies were picked and stained with fluorescent antibodies to evaluate their lineage markers (51).

$\left[{ }^{3} \mathrm{H}\right]$ Thymidine incorporation assay. Bone marrow was isolated and maintained on IL-7-producing NIH3T3 cells as previously described (16). Seven days thereafter, cells were analyzed for the presence of $\mathrm{CD} 19^{+} \mathrm{CD} 43^{+}$cells by FACS. Subsequently, cells were plated in 96 round-bottom wells $\left(2 \times 10^{5}\right.$ cells per well) and stimulated with $10 \mathrm{ng} / \mathrm{ml} \mathrm{IL-7} \mathrm{as} \mathrm{indicated} \mathrm{or} \mathrm{cultivated}$ in the absence of cytokine. $\left[{ }^{3} \mathrm{H}\right]$ Thymidine was added 36 hours thereafter for another 12 hours. Similarly, $2 \times 10^{5}$ tumor cells were plated in 96-well plates; 18 hours thereafter, $\left[{ }^{3} \mathrm{H}\right]$ thymidine was added, and the cells were incubated for another 12 hours (16).

Injection of tumor cells into mice. For s.c. application or application via the tail vein, $10^{6}$ cells from 3 independently derived TYK $2^{--}$and 3 TYK2 ${ }^{+-}$cell lines were resuspended in $300 \mu \mathrm{l}$ PBS and injected into 3 nude mice each. At the time point of injection, the cell lines were analyzed for $\mathrm{CD} 19^{+}$cells by FACS analysis. Nude mice injected with PBS served as controls. Tail vein-injected mice were sacrificed after 29 days. Spleen weights, white blood cell counts, and the presence of $\mathrm{CD} 19^{+}$cells in the bone marrow and blood were analyzed (16). The mice that had received tumor cells s.c. were sacrificed after 12 days, and the tumors were excised and analyzed. 
For s.c. application of EL-4 cells, $10^{5}$ cells were resuspended in $200 \mu \mathrm{l}$ PBS and injected into $6 \mathrm{TYK}^{+/+}$and $6 \mathrm{TYK} 2^{-/-}$mice.

For tumor transplantations, $2 \times 10^{6}$ tumor cells of A-MuLV-infected TYK2 $2^{-/-}$and TYK2 ${ }^{+/-}$mice were injected s.c. into 7 -week-old C57BL/6 mice. Each primary tumor was maintained in culture for 4-5 days to obtain stable tumor cell cultures with high viability $(80 \%)$ and equal proliferative capacity. The cells were then injected into 2 recipient mice each. The secondary tumors were excised 2 weeks after injection and analyzed.

Electrophoretic mobility shift assay. An end-labeled, double-stranded oligonucleotide corresponding to the GAS sequence of the SIEm 67 high-affinity $c$-fos promoter site was used to visualize STAT1- and STAT3-containing DNAbinding complexes. The assay was performed as previously described (52). Antibodies from Santa Cruz Biotechnology Inc. were used to verify DNA binding by supershift analysis (anti-STAT1 [M-22] and anti-STAT3 [C-20]).

FACS analysis. Single-cell suspensions were preincubated with anti-CD16/ CD32 antibodies (BD Biosciences - Pharmingen) to prevent nonspecific $\mathrm{Fc}$ receptor-mediated binding. Antibodies used for lineage determination included B220, CD19, CD43, CD4, CD8, CD3, and NK1.1 (BD Biosciences - Pharmingen). An antibody against MHC class I (clone M1/42.3.9.8.HLK; American Type Culture Collection) was used after labeling with the Alexa Fluor 488 Monoclonal Antibody Kit (Invitrogen Corp.).

Isolation of NK/NKT cells. Freshly isolated splenocytes were incubated with MACS DX5-coupled beads (Miltenyi Biotec GmbH) and subjected to positive selection using the corresponding column system. NK/NKT cells were subsequently cultured in $5,000 \mathrm{U} / \mathrm{ml}$ recombinant human IL-2 for 10 days (53).

Semiquantitative RT-PCR and oligo-bybridization. First-strand cDNA synthesis and PCR amplification were performed with an RT-PCR kit (GeneAmp RNA PCR Kit; PerkinElmer Inc.) according to the manufacturer's instructions. Primer sequences and the procedure used for Southern blotting have been described previously (16).

Cytotoxicity assay. Lactate dehydrogenase release was used to monitor target cell lysis. NK/NKT cells were mixed at the ratios indicated in the figure legends with $2 \times 10^{4}$ YAC- 1 cells per well or with $10^{4}$ tumor-derived cells from A-MuLV-infected TYK2 $2^{-/-}$and $\mathrm{TYK}^{+/-}$mice per well. When YAC-1 cells were the target cells, the incubation lasted for 4 hours, whereas tumor cells were incubated for 8 hours with effector cells. Lactate dehydrogenase release was measured as previously described (54) using the CytoTox 96 Non-Radioactive Cytotoxicity Assay (Promega Corp.).

IFN $-\gamma$ ELISA. In the ELISA for murine IFN- $\gamma, 5 \times 10^{4}$ and $2.5 \times 10^{4} \mathrm{NK} /$ NKT cells were cultured in the presence of $1 \mathrm{ng} / \mathrm{ml} \mathrm{IL}-12$ or of $2,500 \mathrm{U} / \mathrm{ml}$ IL-2, or in the absence of any cytokine (basal condition), in 96-well tissueculture dishes. For each data point, 5 wells were plated and analyzed. After
48 hours, the supernatant was collected, and the concentration of IFN- $\gamma$ was measured by ELISA. For each experiment the NK/NKT cells of 3 mice per genotype were pooled.

MTT assay. NK/NKT cells were seeded in 96-well plates with $5 \times 10^{4}$ cells in $100 \mu \mathrm{l}$ RPMI medium per well. Ten microliters of a $5-\mathrm{mg} / \mathrm{ml}$ stock solution of 3-(4,5-dimethylthiazol-2-yl)-2,5-diphenyltetrazolium bromide (MTT) was added. Four hours thereafter, $100 \mu \mathrm{l}$ stop solution (10\% [wt/ vol] SDS, $50 \%$ [vol/vol] $N, N$-dimethylformamide) was added to solubilize the MTT crystals. The plates were then incubated overnight at $37^{\circ} \mathrm{C}, 5 \%$ $\mathrm{CO}_{2}$, and the OD was read at $620 \mathrm{~nm}$ using an ELISA reader. The values were obtained from 5 individual wells each.

Leukemia formation in TEL-JAK2 transgenic animals. TEL-JAK2 transgenic mice have been described previously (15). Mice ( $\mathrm{F}_{10}$ on a C57BL/ 6 genetic background) were further backcrossed for 6 generations to the C57BL/ 6 background and subsequently intercrossed with TYK2 ${ }^{-/-}$animals. Leukemia development was compared between littermates. The breeding was set up between a TYK2 ${ }^{+/-}$TEL-JAK2 transgene-positive male and TYK2 2 - females.

Statistical analysis. Statistical analysis was carried out using the Student's $t$ test and the $\chi^{2}$ test as appropriate. Kaplan-Meier plots were analyzed for statistical significance using log rank tests.

\section{Acknowledgments}

We thank Udo Losert and the staff of the Biomedical Research Institute for taking good care of our mice. We thank Naomi Rosenberg for the generous gift of A010 cells. We are grateful to Hartmut Beug, Christine Mannhalter, Richard Moriggl, Christian Sillaber, and Peter Valent for helpful discussions. We also thank Thomas Kolbe and Robert Lajko for technical help. This work was supported by grant P15033 from the Fonds zur Förderung der Wissenschaftlichen Forschung (FWF) and grant 2032 from the Vienna Research Council (to V. Sexl), Jubiläumsfondprojekt number 9875 of the Austrian National Bank (to D. Stoiber), FWF grant P15335 (to M. Karaghiosoff), and grant OEZBT GZ200.074/ $\mathrm{I}-\mathrm{VI} / \mathrm{Ia} / 2002$ from the Bundesministerium für Bildung Wissenschaft und Kultur (to M. Muller).

Received for publication June 1, 2004, and accepted in revised form October 4, 2004.

Address correspondence to: Veronika Sexl, Department of Pharmacology, Medical University of Vienna, Waehringerstrasse 13A, A-1090 Vienna, Austria. Phone: 0043-1-4277-64137; Fax: 0043-14277-9641; E-mail: veronika.sexl@meduniwien.ac.at.
1. Ihle, J.N. 2001. The STAT family in cytokine signaling. Curr. Opin. Cell Biol. 13:211-217.

2. O'Shea, J.J., Gadina, M., and Schreiber, R.D. 2002. Cytokine signalling in 2002: new surprises in the Jak/Stat pathway. Cell. 109:121-131.

3. Igaz, P., Toth, S., and Falus, A. 2001. Biological and clinical significance of the JAK-STAT pathway: lessons from knockout mice. Inflamm. Res. 50:435-441.

4. Yu, H., and Jove, R. 2004. The Stats of cancer: new molecular targets come of age. Nat. Rev. Cancer. 4:97-105.

5. Garcia, R., et al. 2001. Constitutive activation of Stat 3 by the Src and JAK tyrosine kinases participates in growth regulation of human breast carcinoma cells. Oncogene. 20:2499-2513.

6. Gao, B., et al. 2001. Constitutive activation of JAKSTAT3 signaling by BRCA1 in human prostate cancer cells. FEBS Lett. 488:179-184.

7. Frank, D.A., Mahajan, S., and Ritz, J. 1997. B lymphocytes from patients with chronic lymphocytic leukemia contain signal transducer and activator of transcription (STAT) 1 and STAT3 constitutively phosphorylated on serine residues. J. Clin. Invest. 100:3140-3148.

8. Coffer, P.J., Koenderman, L., and de Groot, R.P. 2000. The role of STATs in myeloid differentiation and leukaemia. Oncogene. 19:2511-2522.

9. Gouilleux-Gruart, V., et al. 1997. Activated STAT related transcription factors in acute leukaemia. Leuk. Lymphoma. 28:83-88.

10. Bromberg, J.F., et al. 1999. STAT3 as an oncogene. Cell. 98:295-303.

11. Kelly, J.A., et al. 2003. STAT5 synergizes with T cell receptor/antigen stimulation in the development of lymphoblastic lymphoma. J. Exp. Med. 198:79-89.

12. Danial, N.N., et al. 1998. Direct interaction of JAK1 and v-Abl is required for v-Abl-induced activation of STATs and proliferation. Mol. Cell. Biol. 18:6795-6804.

13. Zhang, Y., et al. 2000. Activation of STAT3 in v-Srctransformed fibroblasts requires cooperation of JAK1 kinase activity. J. Biol. Chem. 275:24935-24944.
14. Lacronique, V., et al. 1997. A TEL-JAK2 fusion protein with constitutive kinase activity in human leukemia. Science. 278:1309-1312.

15. Carron, C., et al. 2000. TEL-JAK2 transgenic mice develop T-cell leukemia. Blood. 95:3891-3899.

16. Sexl, V., et al. 2003. JAK1 deficiency leads to enhanced Abelson-induced B-cell tumor formation. Blood. 101:4937-4943.

17. Dunn, G.P., Bruce, A.T., Ikeda, H., Old, L.J., and Schreiber, R.D. 2002. Cancer immunoediting: from immuno-surveillance to tumor escape. Nat. Immunol. 3:991-998.

18. Kaplan, D.H., et al. 1998. Demonstration of an interferon $\gamma$-dependent tumor surveillance system in immunocompetent mice. Proc. Natl. Acad. Sci. U. S. A. 95:7556-7561.

19. Shankaran, V., et al. 2001. IFN $\gamma$ and lymphocytes prevent primary tumour development and shape tumour immunogenicity. Nature. 410:1107-1111.

20. Street, S.E., Cretney, E., and Smyth, M.J. 2001. Perforin an interferon $-\gamma$ activities independently 
control tumor initiation, growth, and metastasis. Blood. 97:192-197.

21. Street, S.E., Trapani, J.A., MacGregor, D., and Smyth, M.J. 2002. Suppression of lymphoma and epithelial malignancies effected by interferon $\gamma$. J. Exp. Med. 196:129-134.

22. Stephanou, A., and Latchman, D.S. 2003. STAT-1: a novel regulator of apoptosis. Int. J. Exp. Pathol. 84:239-244.

23. Velazquez, L., Fellous, M., Stark, G.R., and Pellegrini, S. 1992. A protein tyrosine kinase in the interferon alpha/beta signaling pathway. Cell. 70:313-322

24. Schindler, C. 1999. Cytokines and JAK-STAT signaling. Exp. Cell Res. 253:7-14.

25. Karaghiosoff, M., et al. 2000. Partial impairment of cytokine responses in TYK2-deficient mice. Immunity. 13:549-560.

26. Shimoda, K., et al. 2000. TYK2 plays a restricted role in IFN $\alpha$ signaling, although it is required for IL-12mediated T cell function. Immunity. 13:561-571.

27. Karaghiosoff, M., et al. 2003. Central role for type I interferons and TYK2 in lipopolysaccharide-induced endotoxin shock. Nat. Immunol. 4:471-477.

28. Abelson, H.T., and Rabstein, L.S. 1970. Influence of prednisolone on Moloney leukemogenic virus in BALB-c mice. Cancer Res. 30:2208-2212.

29. Rosenberg, N., and Witte, O.N. 1988. The viral and cellular forms of the Abelson (abl) oncogene. $A d v$. Virus Res. 35:39-81.

30. Faderl, S., et al. 1999. The biology of chronic myeloid leukemia. N. Engl. J. Med. 341:164-172.

31. Specchia, G., et al. 1995. Ph positive acute lymphoblastic leukaemia in adults: molecular and clinical studies. Leuk. Lymphoma. 18:37-42.

32. Peeters, P., et al. 1997. Fusion of TEL, the ETS-variant gene 6 (ETV6), to the receptor-associated kinase JAK2 as a result of $\mathrm{t}(9 ; 12)$ in a lymphoid and $\mathrm{t}(9 ; 15 ; 12)$ in a myeloid leukemia. Blood. 90:2535-2540.

33. Vallera, D.A., Taylor, P.A., Aukerman, S.L., and Blazar, B.R. 1993. Antitumor protection from the murine T-cell leukemia/lymphoma EL4 by the continuous subcutaneous coadministration of recombinant macrophage-colony stimulating factor and interleukin-2. Cancer Res. 53:4273-4280.

34. Visseren, M.J., et al. 1994. Production of interleukin-2 by EL4 tumor cells induces natural killer cell- and T-cell-mediated immunity. J. Immunother. Emphasis Tumor Immunol. 15:119-128.

35. Akamatsu, S., et al. 2002. Antitumor activity of interleukin-18 against the murine T-cell leukemia/ lymphoma EL-4 in syngeneic mice. J. Immunother. 25(Suppl. 1):S28-S34

36. Bowman, T., Garcia, R., Turkson, J., and Jove, R. 2000. STATs in oncogenesis. Oncogene. 19:2474-2488.

37. Xie, S., Lin, H., Sun, T., and Arlinghaus, R.B. 2002 $\mathrm{JAK} 2$ is involved in c-Myc induction by $\mathrm{Bcr}-\mathrm{Abl}$. Oncogene. 21:7137-7146.

38. Lowdell, M.W., Lamb, L., Hoyle, C., Velardi, A., and Prentice, H.G. 2001. Non-MHC-restricted cytotoxic cells: their roles in the control and treatment of leukaemias. Br. J. Haematol. 114:11-24.

39. Street, S.E., et al. 2004. Innate immune surveillance of spontaneous B cell lymphomas by natural killer cells and $\gamma \delta$ T cells. J. Exp. Med. 199:879-884.

40. Baron, F., et al. 2002. Leukemic target susceptibility to natural killer cytotoxicity: relationship with BCR-ABL expression. Blood. 99:2107-2113.

41. Gruber, T.A., Skelton, D.C., and Kohn, D.B. 2002 Requirement for NK cells in CD40 ligand-mediated rejection of Philadelphia chromosome-positive acute lymphoblastic leukemia cells. J. Immunol. 168:73-80.

42. Inngjerdingen, M., Rolstad, B., and Ryan, J.C. 2003. Activating and inhibitory Ly 49 receptors modulate NK cell chemotaxis to CXC chemokine ligand (CXCL) 10 and CXCL12. J. Immunol. 171:2889-2895.

43. Soriano, S.F., et al. 2002. Functional inactivation of CXC chemokine receptor 4-mediated responses through SOCS3 up-regulation. J. Exp. Med. 196:311-321
44. Garcia-Lora, A., Algarra, I., and Garrido, F. 2003. MHC class I antigens, immune surveillance, and tumor immune escape. J. Cell. Physiol. 195:346-355.

45. Jager, E., et al. 1997. Immunoselection in vivo: independent loss of MHC class I and melanocyte differentiation antigen expression in metastatic melanoma. Int. J. Cancer. 71:142-147.

46. Shimoda, K., et al. 2002. Partial impairment of interleukin-12 (IL-12) and IL-18 signaling in TYK2deficient mice. Blood. 99:2094-2099.

47. Kobayashi, M., et al. 1989. Identification and purification of natural killer cell stimulatory factor (NKSF), a cytokine with multiple biologic effects on human lymphocytes. J. Exp. Med. 170:827-845.

48. Magram, J., et al. 1996. IL-12-deficient mice are defective in IFN $\gamma$ production and type 1 cytokine responses. Immunity. 4:471-481.

49. Sexl, V., et al. 2000. STAT5a/b contribute to interleukin 7-induced B-cell precursor expansion, but abl- and bcr/abl-induced transformation are independent of stat5. Blood. 96:2277-2283.

50. Shinkai, Y., et al. 1992. Rag-2-deficient mice lack mature lymphocytes owing to inability to initiate V(D)J rearrangement. Cell. 68:855-867.

51. Szremska, A.P., et al. 2003. JunB inhibits proliferation and transformation in B-lymphoid cells. Blood. 102:4159-4165

52. Stoiber, D., Stockinger, S., Steinlein, P., Kovarik, J., and Decker, T. 2001. Listeria monocytogenes modulates macrophage cytokine responses through STAT serine phosphorylation and the induction of suppressor of cytokine signaling 3. J. Immunol. 166:466-472.

53. Cerwenka, A., et al. 2000. Retinoic acid early inducible genes define a ligand family for the activating NKG2D receptor in mice. Immunity. 12:721-727.

54. Decker, T., and Lohmann Mattes, M.L. 1988. A quick and simple method for the quantitation of lactate dehydrogenase release in measurements of cellular cytotoxicity and tumor necrosis factor (TNF) activity. J. Immunol. Methods. 115:61-69. 